\title{
PROPERTIES OF POLYCYCLIC AROMATIC HYDROCARBONS IN THE NORTHWEST PHOTON DOMINATED REGION OF NGC 7023. I. PAH SIZE, CHARGE, COMPOSITION, AND STRUCTURE DISTRIBUTION
}

\author{
C. Boersma, J. D. Bregman, and L. J. Allamandola \\ NASA Ames Research Center, MS 245-6, Moffett Field, CA 94035-0001, USA; Christiaan.Boersma@nasa.gov \\ Received 2012 September 6; accepted 2013 March 19; published 2013 May 14
}

\begin{abstract}
Polycyclic aromatic hydrocarbon (PAH) emission in the Spitzer Infrared Spectrograph spectral map of the northwest photon dominated region (PDR) in NGC 7023 was analyzed exclusively using PAH spectra from the NASA Ames PAH IR Spectroscopic Database (www.astrochem.org/pahdb). The 5-15 $\mu \mathrm{m}$ spectrum at each pixel is fitted using a non-negative-least-squares fitting approach. The fits are of good quality, allowing decomposition of the PAH emission into four subclasses: size, charge, composition, and hydrogen adjacency (structure). Maps tracing PAH subclass distributions across the region paint a coherent astrophysical picture. Once past some 20 seconds of arc from HD 200775, the emission is dominated by the more stable, large, symmetric, compact PAH cations with smaller, neutral PAHs taking over along the lines-of-sight toward the more distant molecular cloud. The boundary between the PDR and the denser cloud material shows up as a distinct discontinuity in the breakdown maps. Noteworthy is the requirement for PANH cations to fit the bulk of the 6.2 and $11.0 \mu \mathrm{m}$ features and the indication of PAH photo-dehydrogenation and fragmentation close to HD 200775. Decomposition of the spectral maps into "principal" subclass template spectra provides additional insight into the behavior of each subclass. However, the general applicability of this computationally more efficient approach is presently undetermined. This is the first time the spectra of individual PAHs are exclusively used to fit the 5-15 $\mu \mathrm{m}$ region and analyze the spatial behavior of the aromatic infrared bands, providing fundamental, new information about astronomical PAH subpopulations including their dependence on, and response to, changes in local conditions.
\end{abstract}

Key words: astrochemistry - infrared: ISM - ISM: individual objects (NGC 7023) - molecular data techniques: spectroscopic

Online-only material: color figures

\section{INTRODUCTION}

The well-known family of emission features near 3.3, 6.2, 7.6, $7.8,8.6,11.2$ and $12.7 \mu \mathrm{m}$, formerly referred to as the unidentified infrared bands (e.g., Gillett et al. 1973; Sellgren 1984; Geballe et al. 1985; Cohen et al. 1986), are now commonly ascribed to mixtures of polycyclic aromatic hydrocarbons (PAHs), large, chicken-wire-shaped molecules of fused aromatic rings, and related species (e.g., Smith et al. 2007b; Tielens 2008). In space, free floating PAH molecules become highly vibrationally excited upon the absorption of a single photon (ultraviolet-nearinfrared; Draine \& Li 2007; Mattioda et al. 2005). Relaxation occurs through emission of infrared (IR) photons at characteristic wavelengths, leaving the tell-tale spectroscopic fingerprints of aromatic molecules.

NGC 7023, also known as the Iris Nebula, is the reflection nebula (RN) in Cepheus irradiated by the Herbig Be star HD 200775. It is located $430 \mathrm{pc}$ from the Sun (van den Ancker et al. 1997) and is one of the best studied $\mathrm{RNe}$ in the sky (e.g., Moutou et al. 1998; Werner et al. 2004b; Witt et al. 2006; Fleming et al. 2010, and references therein). Most recently, IR emission from NGC 7023 has shown the presence of interstellar $\mathrm{C}_{60}$ in its neutral (Sellgren et al. 2007, 2010) and cationic form $\mathrm{C}_{60}^{+}$(Berné et al. 2013).

NASA's Spitzer Space Telescope (Werner et al. 2004a) obtained a spectral map of the northwest photon dominated region (PDR) of NGC 7023 utilizing the Infrared Spectrograph (IRS; Houck et al. 2004). The low resolution set of these data has been analyzed by Berné et al. (2007) and the high resolution set by Rosenberg et al. (2011), both using blind signal separation (BSS) techniques. Rosenberg et al. (2011) further analyzed the three independent spectral signals resulting from the BSS analysis using the NASA Ames PAH IR Spectroscopic Database (PAHdb; Bauschlicher et al. 2010). Here that work is extended by combining the low and high-resolution observational data and decomposing the spectrum at each map position using PAHdb.

PAHdb is a collection of experimentally measured and theoretically computed PAH spectra. The database, currently at version 1.32 , contains the spectra of nearly $700 \mathrm{PAHs}$ of different shapes, sizes, compositions and ionization states. The data is accessible at www.astrochem.org/pahdb, where it can be downloaded together with a suite of IDL tools (AmesPAHdbIDLSuite), including the fitting procedure used here.

The aim of this paper (Paper I) is to demonstrate how decomposition of the aromatic IR emission features using solely a database comprised of the spectra of individual PAHs, combined with a non-negative-least-squares fit approach, paints a consistent, detailed, and novel picture of how the different PAH subclasses making up the PAH population evolve in response to changing local conditions. This is done in a way not previously possible. For example, as discussed in Section 4, this approach provides information about PAH composition and structure that is not accessible by any other means. Paper II will present a critical comparison between the results derived from the computational analysis used here with those derived from a "classical" analysis of the PAH band features.

This work is presented as follows. The observations and data reduction are discussed in Section 2. Section 3 presents a stepwise description of the spectral analysis, including removal of the broad band continuum and emission lines from the spectra, correcting for extinction, fitting the "clean" spectra, 


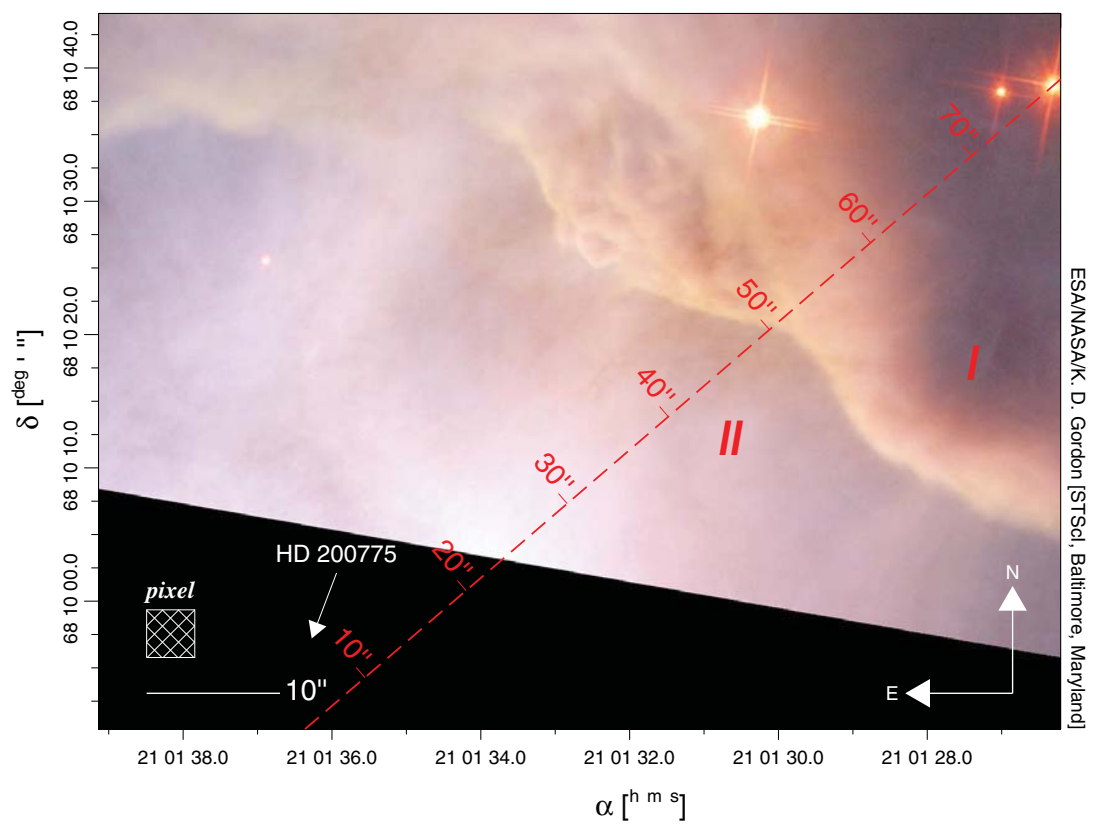

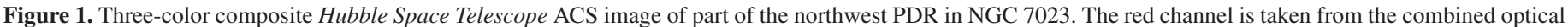

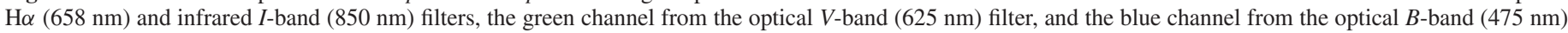

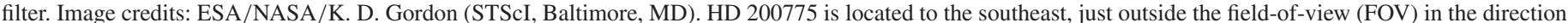

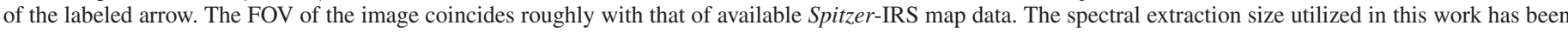

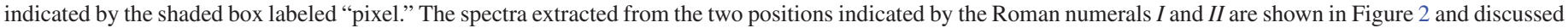
in Section 3. The dashed line runs from HD 200779 to the YSO in the northwest corner of the images, with distance from HD 200775 indicated in seconds of arc.

(A color version of this figure is available in the online journal.)

and decomposing the overall $\mathrm{PAH}$ emission spectrum into contributing subclasses. The results are discussed in Section 4, and Section 5 summarizes and concludes this work.

\section{OBSERVATIONS AND DATA REDUCTION}

Figure 1 presents a Hubble Space Telescope (HST) image of a region of NGC 7023 northwest of HD 200775. This image includes the brightest PDR in NGC 7023, which is located some 40-50 seconds of arc northwest of the star. Three different morphological regimes can be identified: (1) a bright ridge crossing the region almost diagonally, marking the boundary between the (2) PDR and the (3) denser molecular cloud. Three young stellar objects (YSOs) are located in the northwest quadrant of the image and one in the northeast quadrant. A dim arc extending toward the star can be distinguished near the center, with a bright region in the lower-middle part of the image. HD 200775 is located to the southeast ( $\alpha$ : 210136.92 , $\delta:+680947.76$ ), just outside the image's field-of-view (FOV). Its direction has been indicated by the labeled arrow in Figure 1.

The FOV in Figure 1 coincides roughly with the region for which Spitzer-IRS spectral mapping data are available. Those observations were downloaded from the Spitzer Heritage Archive and consist of a short-wavelength-low-resolution (SL) and a short-wavelength-high-resolution ( $\mathrm{SH}$ ) map. The low resolution data cover $5.2-14.5 \mu \mathrm{m}$ at a resolution of $R \equiv$ $\lambda / \Delta \lambda \sim 64-128$ and the high resolution data cover 9.9-19.6 $\mu \mathrm{m}$ at a resolution of $R \sim 600$. The data were reduced using the CUbe Builder for IRS Spectra Maps (CUBISM; Smith et al. 2007a). CUBISM was utilized in its default configuration with the latest available calibration files. The extracted data cubes were written to file separately for SL orders SL1 \& SL2 and SH data, leaving out the SL3 "bonus order." Utilizing the tools available in the IDL Astronomy Library (Landsman 1993), the
SL and SH data were aligned and mapped onto the spatial grid of the SH data. This results in data cubes covering the spatial dimensions with $39 \times 29$ pixels. Note that no spatial corrections were applied for the wavelength dependent pointspread function (PSF). Next, bad pixels were interpolated in the spatial domain. To match, more or less, the pixel size to the PSF, the aligned spectral maps were rebinned from the $39 \times 29$ to a $20 \times 15$ spectral map. A pixel size of $\sim 3.6 \times 3.6$ seconds of $\operatorname{arc}^{2}$ was reached and has been indicated in Figure 1 for reference. Errors are propagated here, and throughout the reduction, by performing the same exercise on the squared uncertainty maps. Spectral orders (SL1 and SL2) and modules (SL and $\mathrm{SH}$ ) were spliced by conserving integrated flux in the spectral region of overlap, while keeping the SL1 data fixed. Uncertainties related to the splicing itself are ignored, but should not exceed $15 \%$. Before merging the data into a single cube, the orders and modules were truncated, keeping the SL data in the region where it overlaps the $\mathrm{SH}$ data. The final 5.2-19.6 $\mu \mathrm{m}$ spectra were rebinned onto a uniformly spaced frequency grid $\left(\Delta v_{k}=5 \mathrm{~cm}^{-1}\right)$ to accommodate the fitting procedures.

Figure 2 presents examples of two of the $300(15 \times 20)$, 5-19.6 $\mu \mathrm{m}$ spectra that sample the region. One records the emission from a shielded region northwest of the PDR (Figure 1, position $I$ ) and the other emission from the diffuse medium between HD 200775 and the PDR (Figure 1, position II). The spectra in Figure 2 show there are clearcut differences in the PAH emission features and plateaus associated with each position. Apart from variations in relative band strengths, underlying plateaus, and underlying continuum, closer inspection also shows differences in peak positions and feature profiles. Figure 3, which presents the spectral changes in the features between 10.8 and $11.8 \mu \mathrm{m}$ occurring along the dashed line in Figure 1, shows the richness and subtleties of these differences as a function of distance from HD 200775. As one 

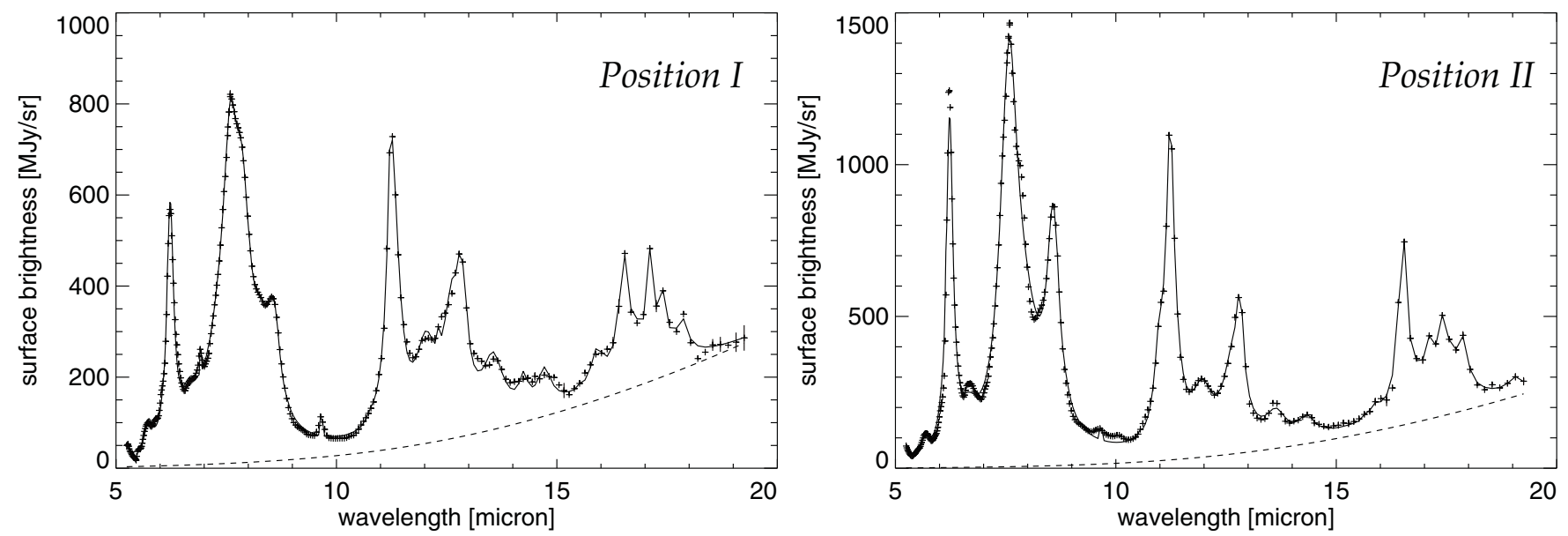

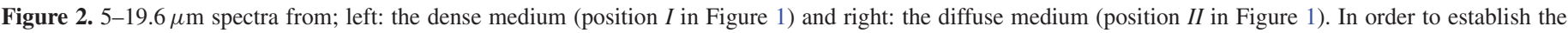

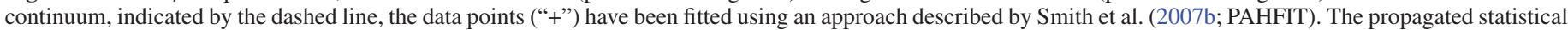
uncertainties, typically smaller than the symbol size, are indicated by vertical lines on top of the data points. See Section 3 for details.

moves from 20 to 60 seconds of arc from the exciting star, there is a steady drop in the ratio of the $11.0-11.2 \mu \mathrm{m}$ band, a reversal in importance of the 11.2 and $11.24 \mu \mathrm{m}$ components of the $11.2 \mu \mathrm{m}$ feature and a subtle increase in the FWHM of the $11.2 \mu \mathrm{m}$ band from $\sim 13$ to $\sim 18 \mathrm{~cm}^{-1}$. There is also evidence of a weak feature peaking near $11.75 \mu \mathrm{m}$ close to the star that redshifts with distance. These aspects will be examined in more detail in Paper II.

\section{ANALYSIS}

To analyze the PAH emission, the continuum component, possible effects from extinction, and hydrogen emission lines must be removed from the spectra. Given the sizable amount of data, an automated approach is used in which the narrow emission lines, broader PAH features, underlying continuum, and extinction are simultaneously modeled and fitted. The emission lines are represented by Gaussian profiles, the PAH features by Drude profiles and the continuum is represented by a power-law/blackbody in Wein's limit. Line and PAH centroids, together with their widths have been taken from Smith et al. (2007b). The centroids are allowed to vary within 15\%, the power of the emission profiles are forced strictly positive, and the maximum blackbody temperature is limited to $100 \mathrm{~K}$. A fully mixed model is assumed for the extinction, which leads to an overall diluting factor of $\left(1-\exp \left(-\tau_{v}\right)\right) / \tau_{v}$ (e.g., Disney et al. 1989; Smith et al. 2007b). The optical depth $\tau_{\nu}$ is in terms of the visual extinction $A_{V}$ (i.e., $\tau_{v}=1.8 \times 10^{21} C_{\text {ext }} A_{V}$ ). The extinction cross-section $\left(C_{\text {ext }}\right)$ used is that of Weingartner \& Draine (2001) for $R_{V}=5.5$. Figure 2 presents the results for the two spectra extracted from the positions labeled $I$ and $I I$ in Figure 1. The figure shows that the approach taken by Smith et al. (2007b) can fit the spectra well.

Next, the emission lines from molecular hydrogen are removed by linearly interpolating across $3 \sigma$ of the feature's width, the spectra are corrected for extinction, and lastly, the derived continua are subtracted from the spectra. Across most of the region extinction is negligible, with the exception in the northwest corner of the region (see Figure 1), where a maximum extinction of $A_{V} \sim 20$ is reached. This is in good agreement with the maximum extinction derived using the $\mathrm{H}_{2}$ lines, where the $S(3)$ over $S(1)$ intensity ratio shows a drop of nearly a factor of three at these positions. Assuming this drop is purely due to extinction and not a change in gas density/temperature, this translates also into a maximum of $A_{V} \sim 20$ (Rieke \& Lebofsky 1985). This value is, given the associated uncertainties and approximations, consistent with those recently determined by Pilleri et al. (2012). Figure 4 presents the results for the two spectra extracted from the same positions as in Figure 2.

To examine the nature of the emitting PAH population, the PAH emission is decomposed using density-functional-theory (DFT) computed spectra from PAHdb utilizing the AmesPAHdbIDLSuite (Bauschlicher et al. 2010). The fits are based on spectra of individual aromatic molecules with specific charge states, structures, compositions, and sizes. This allows the analysis of the spectra without invoking an ad hoc interpretation of the state of the PAH population since the average synthesized spectrum can be traced back to the fully characterized individual PAH molecules.

When comparing astronomical emission spectra to computed PAH absorption spectra, band shape, natural line width, band shifts, and relative band intensities inherent to the emission process must be taken into account. Some discussion of these band properties can be found in, for example, Bauschlicher et al. (2010) and Rosenberg et al. (2011). The approaches taken in modeling the emission from individual PAHs here are summarized below.

Band shift. Since the astronomical emission spectra originate in highly vibrationally excited molecules, anharmonic effects induce a small redshift to the emission band peak positions relative to the band positions measured in absorption. Here a $15 \mathrm{~cm}^{-1}$ redshift is taken, a value consistent with shifts measured in a number of experimental mid-IR studies (Cherchneff \& Barker 1989; Flickinger \& Wdowiak 1990, 1991; Flickinger et al. 1991; Colangeli et al. 1992; Brenner \& Barker 1992; Joblin et al. 1995; Williams \& Leone 1995; Cook \& Saykally 1998). However, all of these experiments have been carried out on species smaller $\left(N_{\mathrm{C}} \leqslant 32\right.$; Joblin et al. 1995) than those that contribute most to the observed mid-IR emission $\left(N_{\mathrm{C}} \sim 50 \lesssim 100\right)$. Unfortunately, the challenges of extending similar experimental studies to a number of large PAHs are daunting, if not insurmountable, making a computational approach attractive. Here too there are significant problems since DFT computations that fully treat anharmonicity in large PAHs are not yet tractable. Furthermore, the degree of anharmonicity is dependent on the excitation level of the molecule and is mode specific. Thus, the single $15 \mathrm{~cm}^{-1}$ redshift is an approximation based on the experimental evidence 


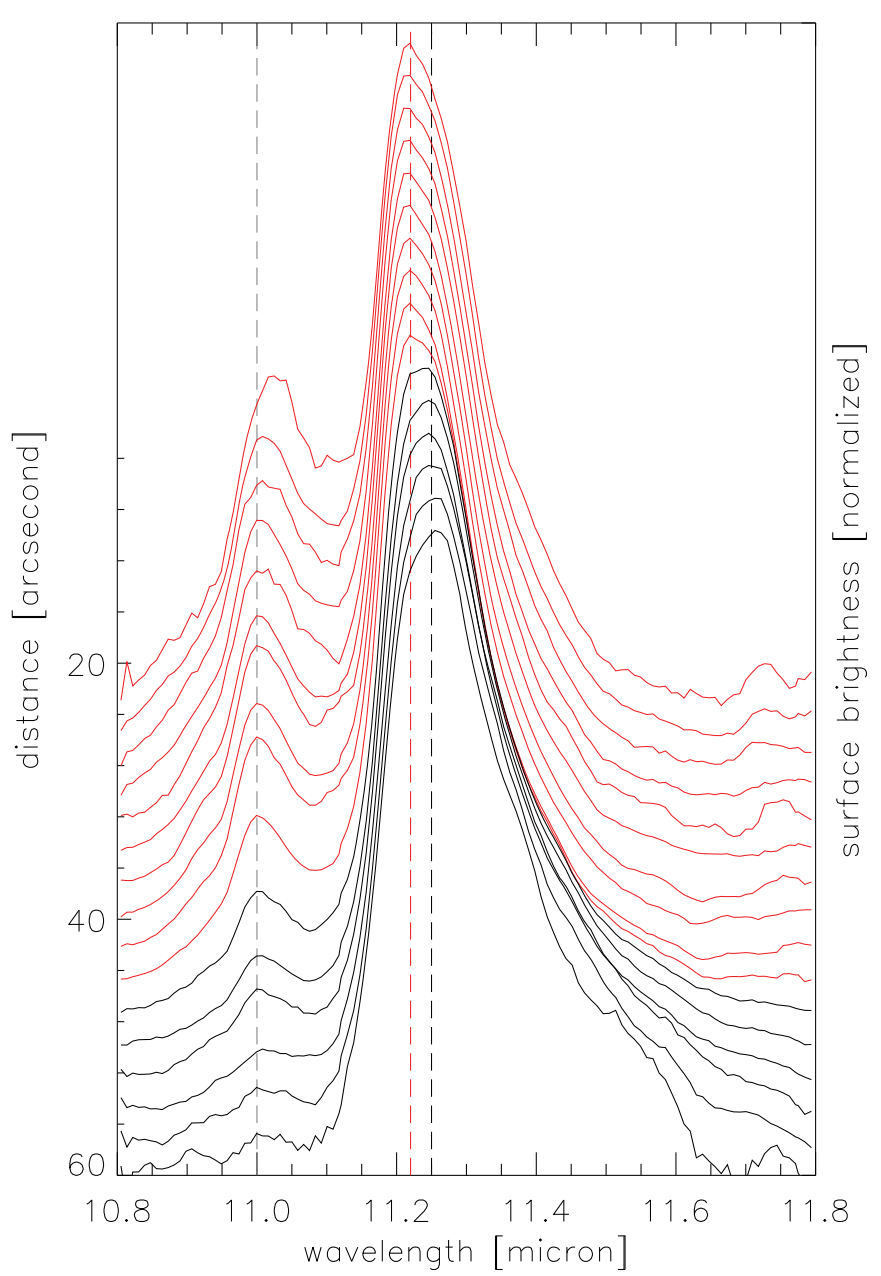

Figure 3. Spectroscopic variations in the $10.8-11.8 \mu \mathrm{m}$ region along the dashed line in Figure 1 from about 20 to 60 seconds of arc from HD 200779. These $\mathrm{SH}$-data, taken at native spectroscopic resolution $(\lambda / \Delta \lambda \sim 600)$, are presented with the underlying continuum linearly interpolated and removed, peak intensity of the feature normalized to one and smoothed over three resolution elements. The dashed vertical lines are centered at 11.0, 11.2 and $11.24 \mu \mathrm{m}$. The spectra shown correspond to 2.5 seconds of arc steps along the dashed line, with the thick (red in the online version of the journal) and thin indicating those sampling the diffuse and dense medium, respectively.

(A color version of this figure is available in the online journal.)

at hand. The effect of varying band shift on the results will be discussed in more detail in a forthcoming paper.

Band profile. Although the intrinsic emission profile for a single, unperturbed vibrational transition is Lorentzian, due to the emission process, the observed bands form from a blend of Lorentzian bands with different centroids and widths (e.g., Pech et al. 2002). The total band profile, both its width and shape, are affected by the diversity in the PAH mixture as well as its range in emission temperatures. Initial testing shows that inherently sharper Gaussian profiles provide a closer fit $\left(\chi^{2} \equiv \sum_{i}^{n}\left(\left(y_{i, \mathrm{obs}}-y_{i, \mathrm{fit}}\right) / y_{i, \mathrm{obs}}\right)^{2}\right)$ to the observed astronomical spectra than Lorentzian or Drude profiles, with the interpretation of the results remaining mostly fixed. From the standpoint of the incomplete database, this is viewed as each species representing a statistical set of species with very similar properties, i.e., peak centroid. The peak positions of the harmonic oscillators contributing to a feature are then distributed randomly around a mean. Indeed, the variations in the observed band profiles, asymmetries, and peak positions evident in Figure 2 and discussed by Peeters et al. (2002) and van Diedenhoven et al. (2004) are consistent with an origin in PAH mixtures in which each individual PAH produces a spectrum comprised of slightly different Lorentzian profiles and intensities. The emission process and anharmonicity effects can also introduce an asymmetry in the emission profile as does blending with overtone, combination, and hot bands (Barker et al. 1987; Pech et al. 2002). These effects are not treated here as they are not yet fully characterized and understood. Pech et al. (2002) provide a description for the former effect, but unfortunately one that is incompatible with the approach taken here. This implies that the fits of the asymmetric red wings of the PAH features must be fully produced by emission from different PAHs in PAHdb. Since PAH anions systematically show emission shifted toward the red compared to their neutral and positively charged counterparts, they are expected to be overrepresented in the final results. For the band width, a value of $15 \mathrm{~cm}^{-1}$ is taken, which is about the average of the observed range in width $\left(\sim 13-18 \mathrm{~cm}^{-1}\right)$ of the $11.2 \mu \mathrm{m}$ features shown in Figure 3. However, as the SH spectra in Figure 3 show, this is clearly wider than the $11.0 \mu \mathrm{m}$ subfeature and may cause some issues, especially between $\sim 10.5$ and $12 \mu \mathrm{m}$. As mentioned earlier, these aspects will be examined in further detail in Paper II.

Emission model. When considering the entire mid-IR emission spectrum from any given $\mathrm{PAH}$, relative band intensities depend on molecule size and average excitation energy. Here the cascade emission model is used, in which each PAH will reach a different maximum temperature that depends on its heat capacity, and stepwise radiative relaxation from that excitation level or temperature is taken into account. The emission model makes use of the thermal approximation (e.g., Verstraete et al. 2001).

Average energy. The exciting star in NGC 7023, HD 200775, is of spectral type B2 (Sellgren 1983; Witt et al. 2006), putting it at an effective temperature of about 22,000 K. Convolving a blackbody spectrum at this temperature with the size-dependent PAH absorption cross-section from Draine \& Li (2007), one finds an average photon energy of $7 \pm 2.5 \mathrm{eV}$. This turns out to be largely insensitive to PAH size and charge.

Although many of the adopted values described above are, by necessity, approximate, they are sound. Furthermore, while the PAH sample in PAHdb is somewhat selection-limited, the database contains significant numbers of species that are representative of the different PAH subclasses, i.e., PAHs that are cationic, neutral, and anionic; large, medium, and small; compact symmetric and non-compact irregular; pure and heteroatom (nitrogen, oxygen, sulfur) containing. Thus, the resulting fits provide new insight into the overall relationship between the chemical and spectroscopic characteristics of the PAH population and the physical conditions across the emitting regions. Specification of the different emission parameters mentioned above (e.g., band shift, band profile, E, etc.) and modeling the actual emission spectra is all handled by the AmesPAHdbIDLSuite. Listing 1, presented in the Appendix, shows example code for fitting a spectrum. It is similar to fit_a_spectrum.pro found in the examples directory of the AmesPAHdbIDLSuite.

It is also important to keep the following six points in mind as one considers the fits to the overall astronomical emission spectra presented below. (1) PAH overtone and combination bands at $5.25 \& 5.7 \mu \mathrm{m}$, and the possible contribution of aliphatic $\mathrm{CH}$ bends in the $6.8 \mu \mathrm{m}$ region, are not represented in the computed spectra of PAHdb. (2) The fit is confined to 

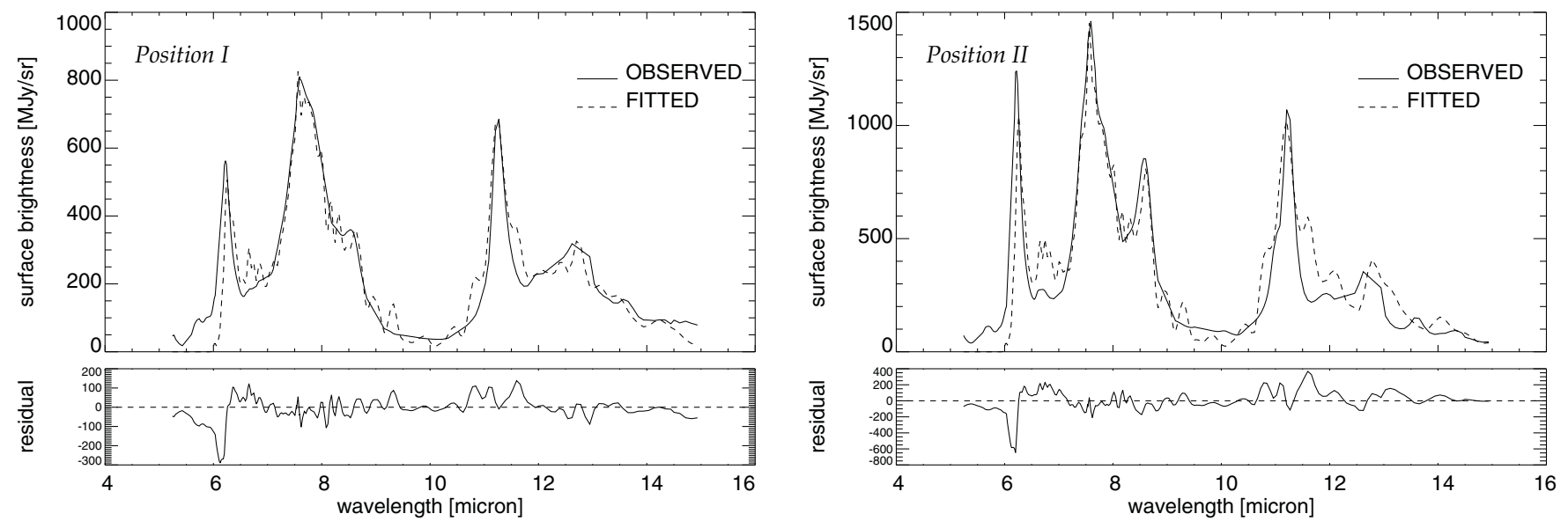

Figure 4. Fit of the 5-15 $\mu \mathrm{m}$ spectra that sample two lines-of-sight toward NGC 7023 with PAHdb. Left: fit of the spectrum from the shielded, dense medium (position $I$ in Figure 1). Right: fit of the spectrum from the radiation rich, diffuse medium (position II in Figure 1). The solid lines are the spectra from Figure 2 extinction corrected and with the emission lines and continuum removed. The dashed lines show the total fit. The lower traces present the residuals produced by subtracting the observed spectrum from the fit. See Section 2 for details on the data reduction and Section 3 for details on the fit.

5-15 $\mu \mathrm{m}$. Boersma et al. (2010) have shown PAHdb lacks the proper species (size and shape) to account for the emission features in the 15-20 $\mu \mathrm{m}$ region. Excluding this region improves the quality of the fit between 5 and $15 \mu \mathrm{m}$ without significantly altering the main results. (3) Fullerene spectra are not yet in PAHdb. Emission from neutral $\mathrm{C}_{60}$ has been detected at 7.0, 8.5, 17.4, and 18.9 $\mu \mathrm{m}$ (Cami et al. 2010; Sellgren et al. 2010, $2007)$ and from its cationic form $\left(\mathrm{C}_{60}^{+}\right)$at $6.4,7.1,8.2$, and $10.5 \mu \mathrm{m}$ (Berné et al. 2013). The 17.4 and $18.9 \mu \mathrm{m}$ fullerene bands fall in the excluded wavelength region, but the others do not. However, the fullerene bands are rather weak and those from $\mathrm{C}_{60}^{+}$only appear very close to the star, therefore, their omission does not adversely affect the fits. DFT calculations on fullerenes are currently underway (C. W. Bauschlicher \& A. Ricca 2013, private communication) and are anticipated to be added to an upcoming version of PAHdb. (4) Only PAHs and PAHs containing nitrogen (PANHs) are included in the fits described here. While only a very limited number of PANH cations are represented in PAHdb, they are included because PANH cations are required to fit the class A $6.2 \mu \mathrm{m}$ band and, as described in Section 3 below, PANHs are required to fit the $11.0 \mu \mathrm{m}$ band as well. Although their presence is currently considered speculative, PANHs may well be important members of the astronomical PAH population since both $\mathrm{CC}$ and $\mathrm{CN}$ containing species-PAH and PANH feedstock-are abundant and intermixed in carbon-rich stellar envelopes, the regions where PAHs are thought to form. Nitrogen incorporation at this stage would be expected. Based on the requirement to match the $6.2 \mu \mathrm{m}$ band, Hudgins et al. (2005) conclude that only $1.2 \%$ of the cosmic nitrogen is locked up in PANHs, where $\sim 20 \%$ of the cosmic carbon is locked up in PAHs (Dwek et al. 1997). A full assessment of the contributions that PANHs may make to the astronomical emission spectra will require the addition of a significant number of computed PANH spectra to the database. While efforts are underway, this is a long-term project. (5) The plateaus underlying the distinct $\mathrm{PAH}$ features are included in the fit. These are generally attributed to emission from aromaticrich materials because of the tight spectroscopic coincidence of these plateaus with the well-known, strong PAH features. The good fits show that, while the fitting procedure used gives no information about the nature of the plateau carrier, the overall trends and the association with aromatic modes are robust. Possible connections between the plateau carriers and very small grains (VSGs) will be explored in greater detail in Paper II. (6) PAHdb lacks spectra from partially to fully dehydrogenated PAHs. Such species would be needed to fit the PAH emission in regions where PAHs are likely to dehydrogenate, i.e., in sufficiently strong radiation fields and/or nearby exciting stars.

Common concerns regarding fitting results are uniqueness and degeneracy. The fits presented here utilize a non-negativeleast-squares approach (Lawson \& Hanson 1974). The nature of mid-infrared emission spectroscopy precludes the identification of specific, individual PAHs since bands from different molecules overlap; rather, it allows one to probe the subclasses, e.g., size, charge, and composition, of the emitting PAH population along a given line-of-sight and to trace their response to changes in their environment. When the spectrum of a specific PAH comprising a fit is removed from the pool of spectra sampled for a subsequent fit, provided there are enough spectra in the pool, another species from the same subgroup will simply replace it. Therefore, a midIR fit made with PAHdb is not expected to provide information about the presence of an individual PAH molecule, but about the subclasses of PAHs that produce the emission spectrum and the response of that subclass to the local astronomical environment. The completeness of PAHdb is of lesser concern, as long as the different subclasses are well represented.

An example showing the quality of the fits is given in Figure 4, which presents fits of the $5-15 \mu \mathrm{m}$ spectra at the two positions indicated in Figure 1 that sample the shielded, dense medium (position $I$ ) and the radiation rich, diffuse medium (position $I I$ ). The figure shows that the fits are of good quality, with some apparent residuals that provide both additional insight into the astronomical PAH population and confidence in the fit. As mentioned above, PAHdb does not include the overtone and combination bands that produce emission between $\sim 5.1$ and $6.1 \mu \mathrm{m}$, nor does it include a large number of PANHs, which are required to produce the class A $6.2 \mu \mathrm{m}$ band. The absence of these transitions in PAHdb are responsible for the notable residual between about 5.1 and $6.1 \mu \mathrm{m}$. The reason for the increase in the residual between roughly 10.5 and $12 \mu \mathrm{m}$ is ascribed to the choice of a profile width $\left(15 \mathrm{~cm}^{-1}\right)$ that is too wide to properly capture the narrow $11.0 \mu \mathrm{m}$ feature. 

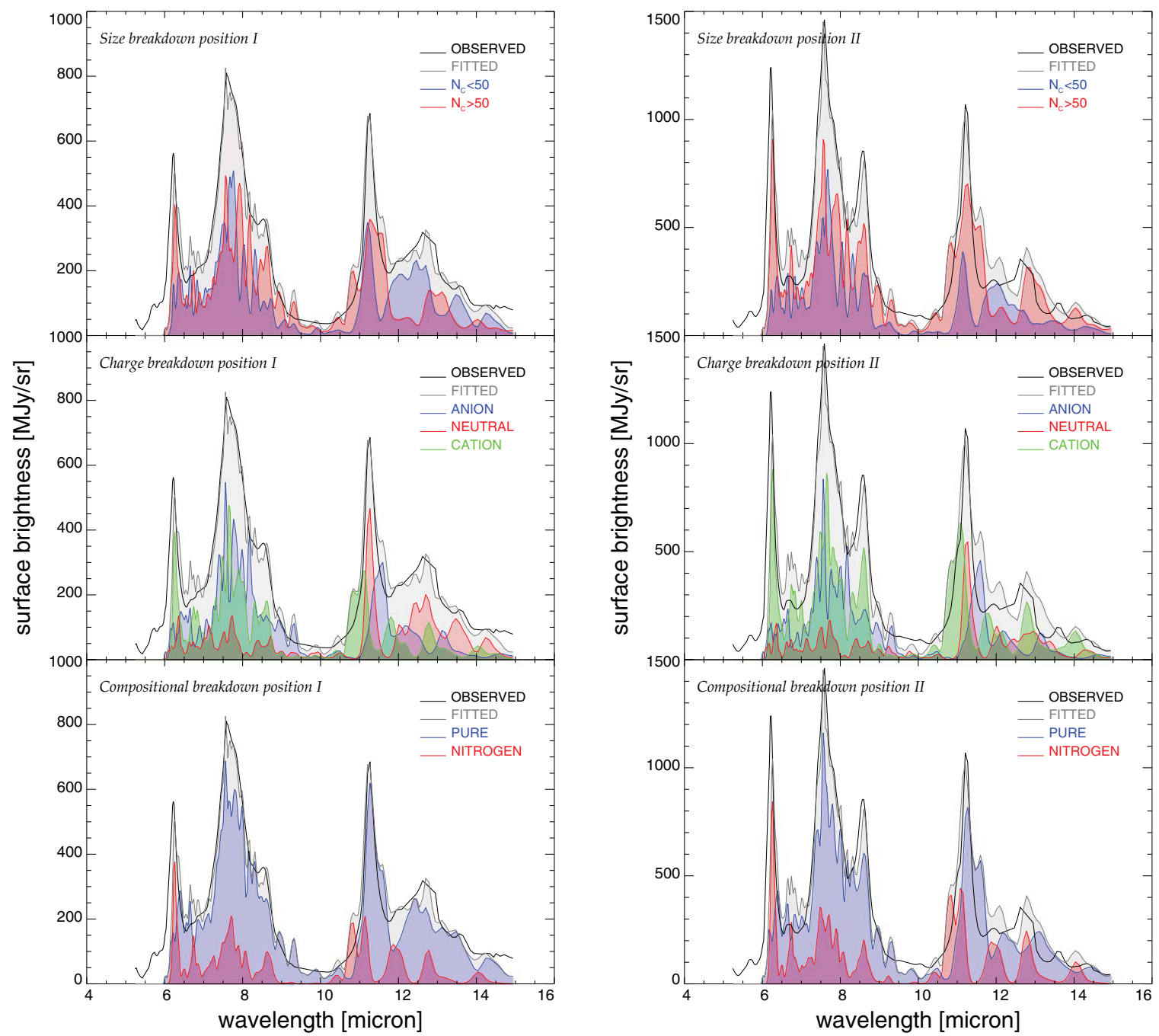

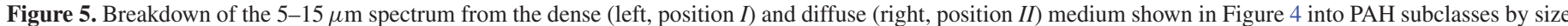
(top), charge (middle), and composition (bottom). See Section 2 for details on the data reduction and Section 3 for details on the fit.
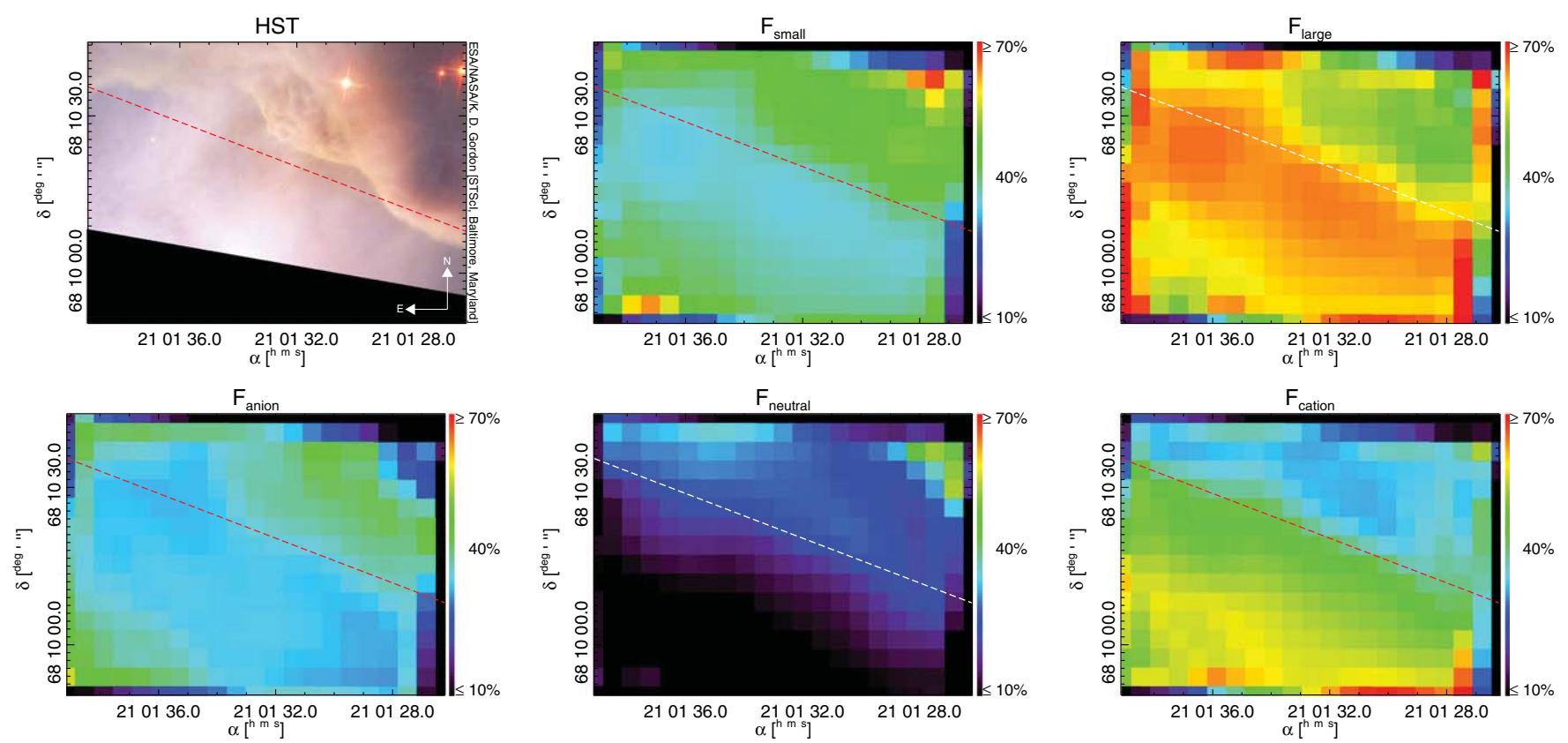

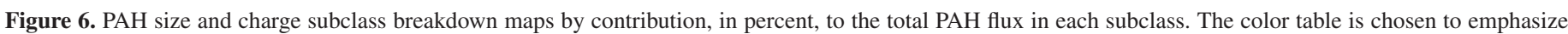

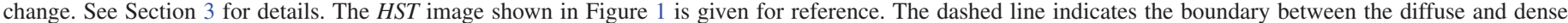
medium across all images as defined by a sudden change in intensity of the $\mathrm{H}_{2} S(1)$ and $S(2)$ emission lines. 
Additionally, as the fits presented in Section 4 indicate that PANHs are also important in this region, their limited number in PAHdb may also contribute. Excluding the 5.1-6.1 $\mu \mathrm{m}$ region, where the data is not fitted by PAHdb, the ratio of the absolute integral of the residuals to the area of the observed spectra at positions $I$ and $I I$ are both $\sim 15 \%$, underscoring the quality of the overall fits.

Figure 5 presents the breakdown of the spectra from positions $I$ and $I I$ shown in Figure 1 into the subclasses; size (split at $N_{\mathrm{C}}=50$ ), charge and composition. These subclass breakdowns are similar to those described in Cami (2011) and Rosenberg et al. (2011). The figure shows global subclass behavior that holds for the fitted spectra at all pixels. In terms of PAH size, the main features and plateaus are filled in about 50/50 by small and large PAHs at position $I$, where at position $I I$ larger PAHs are more prominent. Concerning charge, except for the $11.2 \mu \mathrm{m}$ band which is clearly dominated by neutral PAHs at both positions and the neutral PAH contribution to the minor bands in the $10-15 \mu \mathrm{m}$ region at position $I, \mathrm{PAH}$ cations contribute significantly to the spectra. In addition to $\mathrm{PAH}$ anions filling in the red wings of the 6.2, "7.7," 8.6, 11.2 , and $12.8 \mu \mathrm{m}$ bands, they add to the core of the "7.7" $\mu \mathrm{m}$ feature as well. Regarding composition, PANHs dominate both the 6.2 and $11.0 \mu \mathrm{m}$ emission at both positions as well as adding significantly to the "7.7," 8.6, 12.0, and $12.7 \mu \mathrm{m}$ features. As mentioned above, the residual on the blue side of the $6.2 \mu \mathrm{m}$ feature in Figure 4 may arise from the incompleteness of the PANH spectra in PAHdb, i.e., the lack of other charge states besides the single charged cations in the database. Indeed, it is remarkable that PANHs are called out so prominently to fit both the 6.2 and $11.0 \mu \mathrm{m}$ bands in spite of the fact that they comprise a very small fraction of the PAHs in PAHdb. PANHs are still required to fit the $11.0 \mu \mathrm{m}$ subfeature even when excluding the $6.2 \mu \mathrm{m}$ band from the fit. The sizable residual, of course, could also arise from a choice of too large a redshift (e.g., 15 versus $5 \mathrm{~cm}^{-1}$ ) that is perhaps inappropriate for PANHs or the absence of an aromatic class of species not present in PAHdb. However, the overall good fit and self consistency of these results indicates there is merit in this approach.

Since PAH clusters and hydrogenated amorphous carbon (HAC) particles likely contribute to the plateau emission underlying the main features, it is important to consider these further in light of the fitting approach described above. Plateaulike features are produced by small particles because inter- and intra-molecular interactions within the material perturb the individual bands arising from the fundamental vibrations of the species making up the particles, shifting and broadening them. This results in band "smearing" across the regions normally associated with the fundamental modes (see, e.g., Morterra \& Low 1983; Lee \& Wdowiak 1993; Colangeli et al. 1995; Baratta et al. 1996; Schnaiter et al. 1998; Mennella et al. 1999, 2003; Furton et al. 1999; Hu \& Duley 2007; Jones 2012a, 2012b, 2012c). If the particles are small enough, they produce broad emission features in the regions of the fundamental vibrational modes and thus are likely contributors to the plateaus that underly the well-known emission features. Despite the absence of spectra of small particles in the database, satisfactory fits result. It is important to note that the fits for these underlying plateaus have a significant contribution from the smaller PAHs. Because larger PAHs tend to have compact, symmetric shapes, their structural units (or chemical subgroups) are repetitive. Consequently, they produce spectra with the major bands falling in narrow wavelength ranges. On the other hand, because smaller PAHs contain many different PAH geometries and types of edge structures, their chemical subgroups are not repetitive. This diversity of subgroups in each PAH produces spectra with bands spanning the wavelength regime characteristic of the particular type of aromatic vibrational mode. Therefore, smaller PAHs can fill out the entire wavelength range of the underlying plateaus. It is plausible that plateau emission is produced by carriers that contain mixtures of PAHs with structures similar to those of the smaller PAHs in PAHdb. Given the requirement of small size for mid-IR emission, VSGs probably contribute to the plateaus (Rapacioli et al. 2005; Berné et al. 2007) and because of the tight spectroscopic coincidence of the plateaus with the well-known PAH features, they must be aromatic-rich.

\section{RESULTS AND DISCUSSION}

\subsection{PAH Subclass Breakdown Maps}

Following the procedure described above for all 300 spectra allows one to produce maps of the region for emission from each of the PAH subclasses. Figure 6 shows the maps for PAH size and charge. The HST image from Figure 1 is included and the dashed lines indicate the border of the PDR front with the diffuse medium, as defined by a sharp increase in the intensity of the $\mathrm{H}_{2}$ $S(1)$ line at $17.0 \mu \mathrm{m}$ and the $\mathrm{H}_{2} S(2)$ line at $12.3 \mu \mathrm{m}$. The PANH distribution map is not shown because it is deemed less reliable than the others due to the lack of anionic and neutral PANHs in the version of PAHdb used here, i.e., the PANH data set is too limited to meet the mapping criteria defined in Section 3. The maps show the contribution to the total PAH flux in a subclass, in percent, using a color scale that enhances differences and runs from $10 \%$ to $70 \%$.

The small and large PAH maps show a clear bisection very close to the interface of the diffuse and dense media as delineated by the change in $\mathrm{H}_{2}$ emission line intensities. Furthermore, in the southeast corner of the map the small PAH fraction gradually increases approaching the exciting star, while the large PAH fraction slightly decreases. This is explained by PAH fragmentation and dehydrogenation driven by the increasing radiation field approaching the star. Due to the limited amount of large irregular PAHs and the absence of dehydrogenated PAHs in PAHdb, the fit relies on smaller PAHs to produce the different PAH edge structures and hydrogen adjacency classes required by the spectra close to the exciting star. Hydrogen adjacency is the number of solo, duo, trio, and quartet hydrogens on the peripheral hexagonal rings for any given $\mathrm{PAH}$ and is a measure of PAH edge structure.

The PAH neutral and cation maps show a bisection that is similar to that in the PAH size maps. As expected, emission from PAH cations dominates the region closest to the star, i.e., the diffuse medium, while emission from neutral PAHs becomes increasingly important across the shielded, dense medium. The presence of PAH anions is fairly high across most of the region, with higher contributions close to the star and in the dense medium. The implication of high anion abundances across the entire region is likely an artifact as anions are needed to reproduce the red wings of many of the features (see Section 3). Recall that the red emission wings may include contributions from transitions which are neglected in the model. Although the presence of PAH anions in space has been suggested (e.g., Bregman \& Temi 2005; Bauschlicher et al. 2009), as described in Section 3, proper inclusion of anharmonic effects in the PAH emission model used would red-shade the bands without the need to invoke $\mathrm{PAH}$ anions to produce the entire 

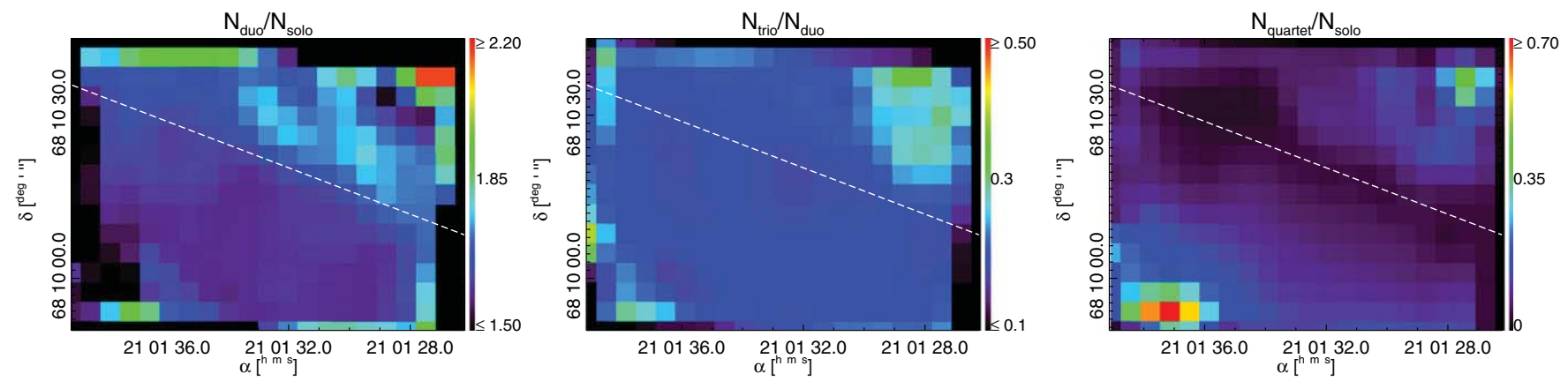

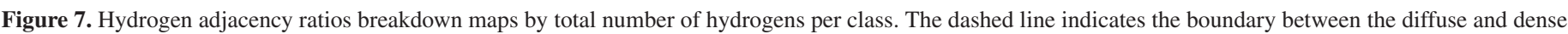
medium across all images as defined by a sudden change in the intensity of the $\mathrm{H}_{2} S(1)$ and $S(2)$ emission lines.

(A color version of this figure is available in the online journal.)

red wing. On the other hand, the spectral breakdowns shown in Figure 5 indicate that PAH anions also contribute to the core of the $6-10 \mu \mathrm{m}$ band complex. At best, this analysis presents an ambiguous case for the presence of PAH anions across the region. This conundrum will likely remain until a far better understanding is achieved of how emission associated with overtone and combination transitions distorts the individual emission bands.

PAH hydrogen adjacency class ratio maps are shown in Figure 7. The $\mathrm{C}-\mathrm{H}$ out-of-plane $\left(\mathrm{CH}_{\mathrm{oop}}\right)$ bending modes of the peripheral hydrogens are responsible for most of the emission between 10 and $15 \mu \mathrm{m}$. Since $\mathrm{CH}_{\text {oop }}$ band peak positions for small PAHs generally fall in narrow ranges that are associated with their hydrogen adjacency class, ratios of observed band strengths in this wavelength region have been used to gain insight into the average PAH edge structure. The average PAH edge structure can then be generalized into the overall PAH shape, as solo hydrogens are commonly associated with long straight edges in the larger, compact, more stable PAH molecules while duo and trio hydrogens are commonly associated with smaller, irregular-shaped PAHs (Bauschlicher et al. 2009; Boersma et al. 2012, and references therein). However, $\mathrm{CH}_{\text {oop }}$ fundamental wavelength variation within a given hydrogen adjacency class results in band overlap, leading to uncertainties in these conclusions (Hudgins \& Allamandola 1999; Hony et al. 2001; Bauschlicher et al. 2008, 2009; Boersma et al. 2009; Ricca et al. 2012). Such uncertainties are significantly reduced with the ability of the fitting procedure to track the characteristics of each individual PAH molecule. As, for the first time, this hydrogen adjacency class information is inherently part of the results. The maps shown in Figure 7 are deduced from the number of hydrogens in each adjacency class from the molecules comprising the fit at each location. That is, the number of hydrogens in each adjacency class for each contributing $\mathrm{PAH}$ is derived by simply counting its number of solo, duo, trio, and quartet hydrogens, multiplying that by its weight retrieved from the fit and finally summing all the hydrogens in each adjacency class.

Figure 7 shows the maps of the duo/solo, trio/duo, and quartet/solo adjacency class ratio distributions. The duo/solo ratio can be interpreted as the ratio of the number of corners over the number of straight edges, the trio/duo ratio can be interpreted as a the ratio of the number of rough corners over smooth corners, and the quartet/solo ratio as the ratio of the number of dangling aromatic rings over the number of straight edges. The maps also show a bisection along the boundary between the diffuse and denser material as well as sharp increases approaching both the exciting star and YSOs.

\subsection{Comparison of PAH Subclass Breakdown Maps with Other Work}

Fleming et al. (2010) have produced maps of the same region using the PAHFIT IDL routines (Smith et al. 2007b) to analyze low resolution 5-14 $\mu \mathrm{m}$ Spitzer IRS spectra. PAHFIT uses Drude profiles to blindly fit the broad, generic PAH features. In their Figure 4, Fleming et al. (2010) show maps of $I_{7.7 \mu \mathrm{m}} / I_{11.3 \mu \mathrm{m}}$ (panel c) and $I_{12.7 \mu \mathrm{m}} / I_{11.3 \mu \mathrm{m}}$ (panel d). While these are not one-to-one tracers of the subclasses mapped here, the similarities and differences merit some discussion. The $7.7 \mu \mathrm{m}$ and $11.3 \mu \mathrm{m}$ bands are often taken as tracers of the $\mathrm{PAH}$ cation and neutral populations, respectively. While this is a reasonable assumption as Fleming et al. (2010) summarize, the maps in Figure 6 above show that neutral and cationic PAH emission not only shifts in going from the shielded, denser region into the diffuse medium but also that emission from all PAH ionization states contribute at all locations. Comparison of their map of $I_{7.7} / I_{11.3}$ with the PAH neutral and cation maps in Figure 6 reveals similar overall behavior. Shifting to the $11.3 \mu \mathrm{m}$ and $12.7 \mu \mathrm{m}$ bands, as discussed above, these can be used as tracers of PAH edge structure, size and overall geometry. Comparison of their map of $I_{12.7} / I_{11.3}$ with the size breakdown maps in Figure 6 again shows similar overall behavior, particularly with the small PAH distribution. Likewise, comparing their $I_{12.7} / I_{11.3}$ map with the hydrogen adjacency class ratio maps shows the greatest similarity with the $N_{\text {trio }} / N_{\text {duo }}$ map. This follows because smaller, less robust PAHs generally have irregular edge structures, i.e., there are more duo and trio hydrogens than solo and duo hydrogens respectively than do the larger, more stable PAHs, which have far more solo hydrogens than any other types. Fleming et al. (2010) also note that the changes in hydrogen adjacency classes can be an indicator of dehydrogenation. Dehydrogenation would hold in regions where the radiation field is strong enough to photo-fragment PAHs and is expected when sampling material in the vicinity of the exciting stars. Because the detailed photo-physics of UV-driven PAH dehydrogenation and fragmentation are unknown, the effect of these processes on the different hydrogen adjacency class ratios is difficult to predict. Furthermore, Hony et al. (2001) point out that while a changing 12.7/11.2 ratio may imply dehydrogenation, this should then go together with, for example, an increasing 6.2/11.2 ratio. However, as discussed by Boersma et al. (2012) for the region within a few minutes of arc south of the ionization ridge in Orion, PAH dehydrogenation and photo-fragmentation scramble hydrogen adjacency classes, a sure sign of significant change in the emitting PAH population. 
Berné et al. (2007) and Rosenberg et al. (2011) have studied the Spitzer spectral maps of NGC 7023 using BSS techniques. They found three principal emission signals that they attribute to PAH cations, neutral PAHs, and VSGs. It is worth noting that the BSS method mainly captures charge state and thus has little to say about PAH sizes and other subclasses. Figure 3 of Rosenberg et al. (2011) presents the spatial distributions for the three BSS signals. Their separation of PAH cations and neutral PAHs (left and central panels, respectively) is consistent with that found here. The component attributed to VSGs (right panel) matches the distribution of small PAHs coincident with the dense medium best. Their VSG distribution does not increase again close to the star as is found here for the small PAH distribution. As explained in Section 4.1, the rise in the smaller PAH population close to the star can, in part, be attributed to the absence of the spectra of dehydrogenated and fragmented PAHs in PAHdb. These species should be prevalent in the region closest to the star. Of course, PAH dehydrogenation and fragmentation does produce smaller PAHs. Although the exact nature of VSGs is not known, as described above, the variable band positions for the fundamental vibrational modes in smaller PAHs can form a basis set that covers the required VSG wavelength range. Rosenberg et al. (2011) also make use of PAHdb to further analyze the three separate components resulting from the BSS breakdown and show both approaches provide a consistent interpretation.

Berné \& Tielens (2012) discuss the formation of $\mathrm{C}_{60}$ close to HD 200775 in NGC 7023. These authors show how the formation of $\mathrm{C}_{60}$ in the proximity of the star could naturally arise from $\mathrm{PAH}$ photo-dehydrogenation, fragmentation and excitation processes driven by the steadily increasing intensity of the UV field. Their models suggest that PAHs containing substantially more than 70 carbon atoms will survive closest to the star, while PAHs containing up to 70 carbon atoms are unstable to graphene formation. Smaller PAHs survive further out, but will be completely dehydrogenated. The hydrogen adjacency maps presented here in Figure 7 are consistent with this overall picture, where all the hydrogen adjacency ratios increase close to the star. In particular, the sharp jump in the quartet/solo ratio in the vicinity of HD 200775 is strong evidence that irregular PAHs take over-as expected from PAH dehydrogenation and fragmentation into smaller species. Simultaneously with the work reported here, Montillaud et al. (2013) modeled the spatial evolution of PAH charge and dehydrogenation in NGC 7023 and find indeed that PAHs quickly dehydrogenate in the region where fullerene is detected.

An example of the changes in $\mathrm{PAH}$ subpopulations as a function of local conditions is shown in Figure 8. The figure tracks the charge and size subclass variations as a function of distance from the exciting star, HD 200775, along the dashed line in Figure 1. The transition from the diffuse to the dense medium is clearly visible around 50 seconds of arc. The transition derived from the maps is broader than that in Figure 1, which is attributed to pixelation. Once beyond $\sim 25$ seconds of arc from the star, the emission fraction from each subclass varies smoothly up to the boundary, behavior consistent with extinction playing little or no role in the excitation process across the diffuse region. This suggests the following overall picture concerning PAH size changes. Along lines-ofsight that probe the increasingly shielded, denser medium, the emission from smaller PAHs increases. This occurs because, in the same radiation field, smaller PAHs are more highly vibrationally excited than are the larger PAHs and the radiation field is not intense enough to destroy them. Moving into the diffuse

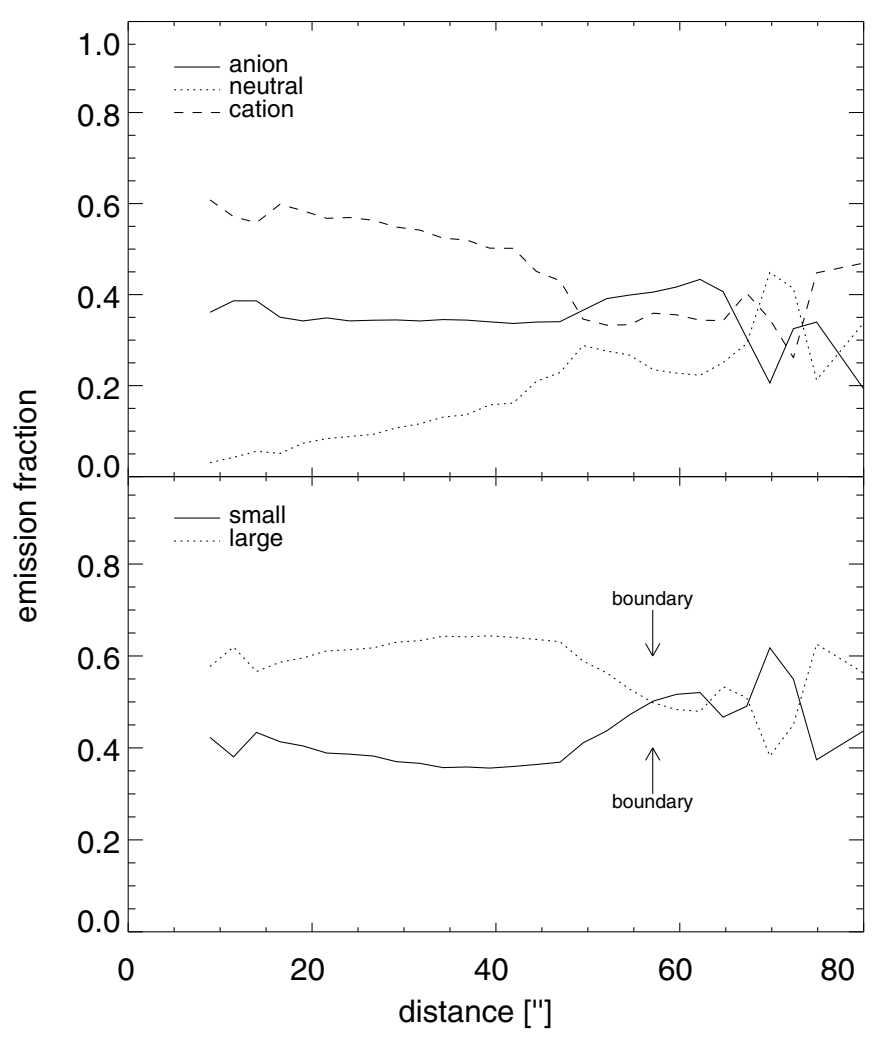

Figure 8. Variations in fractional emission from the PAH subclasses charge (top) and size (bottom) along the dashed line shown in Figure 1. Distances from the exciting star, HD 200775, are in seconds of arc. The double arrow between 50 and 60 seconds of arc indicates the position of the boundary between the dense and diffuse medium as defined by the sudden change in intensity of the $\mathrm{H}_{2} S(1)$ and $S(2)$ emission lines.

medium, exposure to the harder and more intense radiation increases the chance of multi-photon events that both whittles out the smallest PAHs and more highly excites the larger, more robust PAHs, shifting the dominance to the large PAHs. Closing in on HD 200775, there comes a point where the larger PAHs are dehydrogenated and start to fragment. This behavior suggests that the radiation field within 25 seconds of arc of the star is sufficient to dehydrogenate and break the larger PAHs (and perhaps VSGs) into smaller PAH fragments. The quartet to solo hydrogen adjacency class ratio map shown in Figure 7 is consistent with this explanation. This is also similar to the behavior reported by Sellgren et al. (2007), whose long-slit spectral studies of NGC 7023 showed the PAH bands dropping within 20 seconds of arc of HD 20075 while the $18.9 \mu \mathrm{m}$ band (now known to be a fullerene band; Cami et al. 2010; Sellgren et al. 2010) increases and peaks in the vicinity of the star. Regarding charge, although there is some variation, cation emission is always dominant across the 25 seconds of arc region closest to the star. The smooth behavior across the diffuse region is in sharp contrast with that past the boundary where the emission fractions fluctuate significantly as the spectra sample the darker parts of the image. The substructure seen beyond 50 seconds of arc matches up with some of the morphological substructure seen in the northwest quadrant of Figure 1. This likely arises from changes in column density. Note, in particular, the sharp change at 70 seconds of arc in all subclass populations which corresponds with the brightening of the region surrounding the YSOs. The relative contribution of PAH cations starts to increase at this point, eventually overtaking emission from the neutral fraction at the YSOs. Concerning size, there is a sudden 


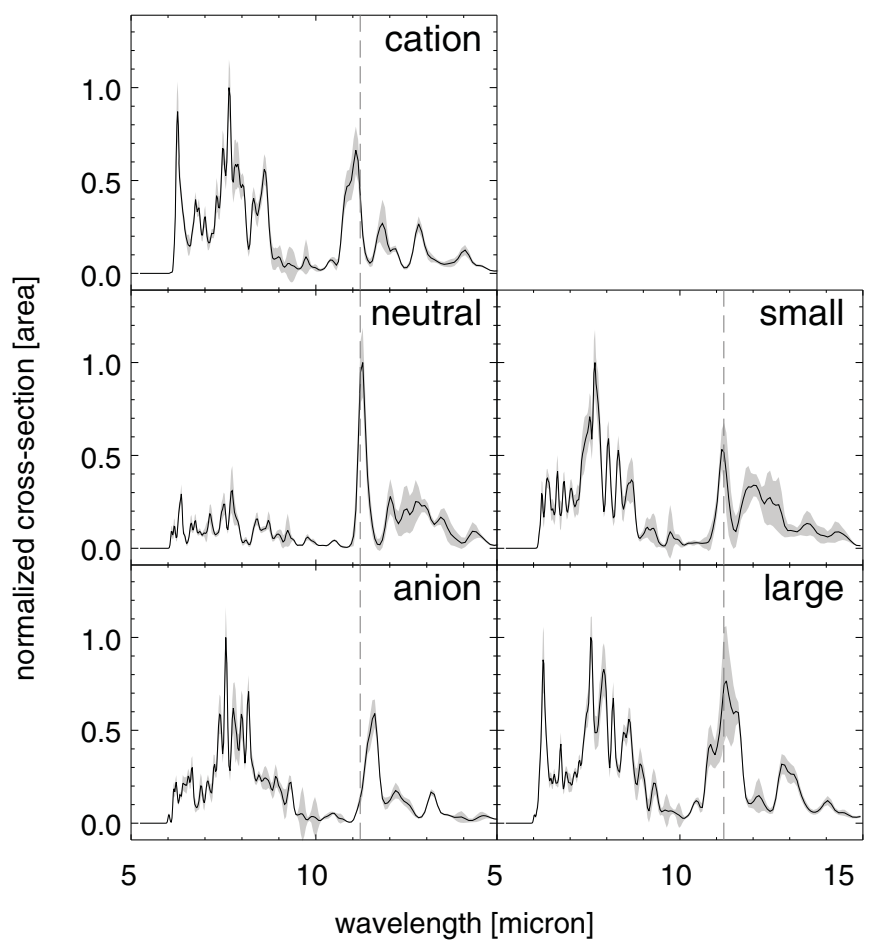

Figure 9. Mean, template spectra for the subclasses by size and charge, with the $1 \sigma$ standard deviation shown as the gray envelopes. The spectrum at each position has been normalized to its peak emission strength. The dashed line centered at $11.2 \mu \mathrm{m}$ highlights the shift in peak position of the $11.2 \mu \mathrm{m} \mathrm{PAH}$ feature. See Section 4 for details.

swap from large to small PAHs dominating the emission at the boundary between the diffuse and dense medium (between 50 and 60 seconds of arc). Apart from the transition across these boundaries, the traces are relatively flat, behavior consistent with stochastic excitation.

\subsection{Template Spectra}

Template spectra for the size and charge subclasses are presented in Figure 9. These templates are the average spectra for each subclass, normalized to their peak intensity. For the average, the integrated flux of each of the (five) subclass spectra at each pixel (300) was normalized to unity. The average and accompanying $1 \sigma$ variation are simply taken over each of the subclasses. The gray envelopes in the figure trace the $1 \sigma$ variation around the average. Constructing the template spectra this way provides insight into the presence of principal emission components whose contribution to the total observed astronomical spectra changes across the nebula. For example, considering the charge subclass templates, the observed spectrum at each position is simply a weighted sum of the three individual templates, $I_{\mathrm{obs}}=a I_{\mathrm{PAH}^{-}}+b I_{\mathrm{PAH}^{0}}+c I_{\mathrm{PAH}^{+}}$.

The templates are consistent with the overall assignments of the different emission components found in the literature, namely that emission in the $5-10 \mu \mathrm{m}$ region is dominated by $\mathrm{PAH}$ cations while that in the $10-15$ region is distributed almost evenly between cationic and neutral PAHs. Furthermore, looking at the peak position and width of the $11.2 \mu \mathrm{m}$ feature demonstrates that the neutral PAH band is flanked to the blue by the PAH cation feature and to the red by the anion band. The biggest differences are associated with the plateaus. Turning to the size template spectra, while they suggest that the $10.5-14 \mu \mathrm{m}$ plateau is indeed largely produced by small PAHs, this is not exclusively the case for the $6-9 \mu \mathrm{m}$ region. Here both the small and large PAH template spectra contain a plateau and strong "narrow" features. While there are several possible "rationalizations" for this spread, as discussed in Section 3, it is likely due to the poor constraints on emission band width and profile which tend to redshift the bands. As also mentioned earlier, since PAH anion bands are generally slightly redshifted with respect to their neutral and cationic counterparts, the fitting procedure oversamples PAH anions. Consequently, the 5-10 $\mu \mathrm{m}$ pedestal in the large PAH template may include an inflated contribution from PAH anions.

The small $1 \sigma$ variation in these template spectra suggests a stable PAH population, where the changing environment primarily changes the relative contribution of each subclass rather than the individual PAHs making up that subclass. The use of these template spectra to break down the spectral map from NGC 7023 into charge subclasses is demonstrated in Figure 10. For comparison purposes the maps are presented on the same scale and with the same color table as in Figure 6. While the maps derived from the template approach are less well defined than those from the fits shown in Figure 6, both figures show good agreement, with the template fit approach carrying far less computational cost than the former approach. However, one does lose the ability to determine hydrogen adjacency classes.

The template spectra are available for download at www.astrochem.org/pahdb/templates. However, the universality of these templates must be further assessed. To this end these template spectra will be compared with those derived from maps of the RN NGC 2023 in a forthcoming paper.

The template spectra derived here can be compared with the BSS extracted spectra from Berné et al. (2007) and Rosenberg et al. (2011), albeit with more spectroscopic detail. The first authors show their three BSS extracted signals in Figure 4 and the second authors in their Figure 3, where the latter only covers $10-15 \mu \mathrm{m}$. The intercomparison of the BSS extracted signals are consistent after taking the effects of differences in bandwidth and spectral and spatial resolution into account (Rosenberg et al. 2011). Berné et al. (2007) attribute signals 1, 2 and 3 resulting from the BSS breakdown to VSGs, PAH neutrals and PAH cations, respectively. It should be noted that these authors do not subtract a broad band continuum prior to their BSS analysis and that they attribute this continuum in the dense medium to VSGs.

There is reasonable resemblance between the average neutral and cation PAH template spectra found here and those retrieved using BSS. For example, the agreement of the overall emission spectra for the neutral PAHs concentrated between 10 and $15 \mu \mathrm{m}$ and that for the PAH cations between 5 and $10 \mu \mathrm{m}$ derived from both methods is good. It is likely that the separation of the VSGs as a distinct component by the BSS approach influences some of the differences that are seen.

Recently, Pilleri et al. (2012), deriving template spectra from the BSS approach, decomposed astronomical spectra into four components: small $\mathrm{PAH}$ cations, small $\mathrm{PAH}$ neutrals, evaporating VSGs (eVSGs), and large ionized PAHs. Their Figure 4 presents the intensity maps for the small PAH cations $\left(\mathrm{PAH}^{+}\right)$, small PAH neutrals $\left(\mathrm{PAH}^{0}\right)$, and $\mathrm{eVSG}$ s for the region studied here. Again, there is qualitative agreement with the results found here. The large-scale structure seen in their $\mathrm{PAH}^{+}$ and $\mathrm{PAH}^{0}$ maps is comparable to that found for the $\mathrm{PAH}$ cation and neutral maps presented here in Figure 6. These authors are, however, able to produce more substructure due to their use of the oversampled Spitzer data products. The spatial distribution of the eVSG component matches up well with the small PAH 

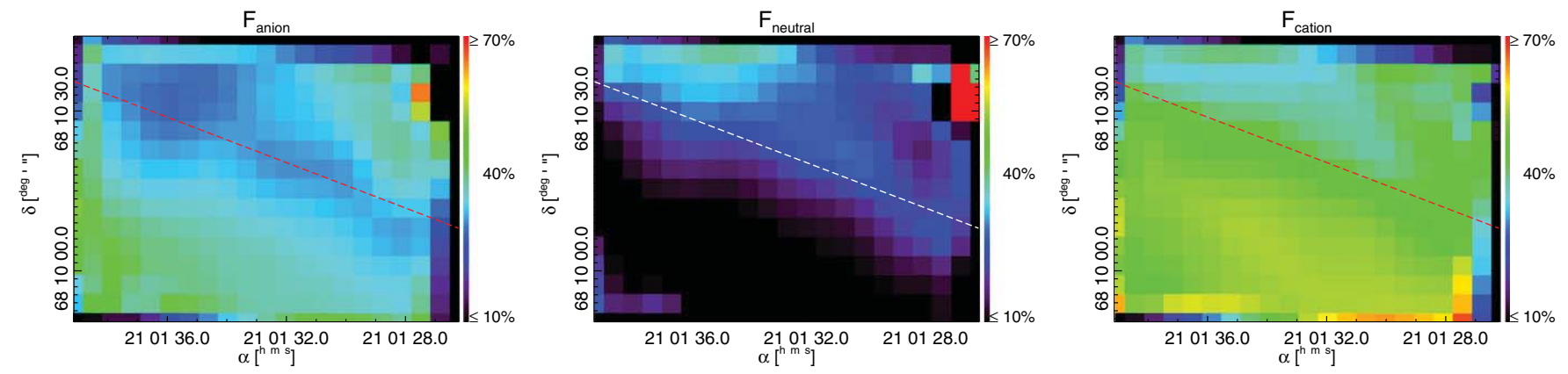

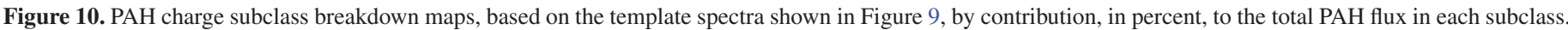

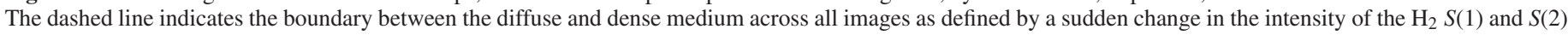
emission lines.

distribution presented here, including similar substructure in the southeast corner of the region close to the star. As described above, this is consistent with the picture of both small and large PAHs evaporating from the grains at the cloud boundary, with emission from small PAHs being strongest in the more distant shielded, denser region (see also Abergel et al. 2002). Again, as discussed above, the increase in eVSGs and small PAHs approaching the exciting star is attributed to large PAH photo-fragmentation driven by a steadily increasing number of multi-photon events.

\section{SUMMARY \& CONCLUSIONS}

The PAH emission in the Spitzer-IRS spectral map of the northwest PDR in NGC 7023 was analyzed using the PAH spectra collected in the NASA Ames PAH IR Spectroscopic Database. This analysis of the aromatic infrared emission bands using the spectra of individual PAHs is novel and provides new, fundamental information about the astronomical PAH family and how it responds to changing local conditions.

After applying a correction for extinction, continuum subtraction and removal of $\mathrm{H}_{2}$ lines, a non-negative-least-squares fit approach was utilized to decompose the PAH emission at each pixel into four subclasses; size, charge, composition, and hydrogen adjacency class. The overall quality of the fits are good. PANHs are required to fit the bulk of both the 6.2 and $11.0 \mu \mathrm{m}$ features. However, even without including the $6.2 \mu \mathrm{m}$ feature, PANHs are required to fit the observed $11.0 \mu \mathrm{m}$ band. The fit of the $6.2 \mu \mathrm{m}$ astronomical PAH feature shows some residual substructure, which is attributed to the limited number of PANHs in PAHdb. To date, nitrogenated PAHs are the only class of PAHs known to have a high enough CC stretching frequency to account for the class A $6.2 \mu \mathrm{m}$ band (Hudgins et al. 2005). A full assessment of the role PANHs might play in producing the overall emission spectra requires the addition of a significant number of PANH spectra to PAHdb.

Maps of PAH subclass distributions across the region paint a coherent astronomical picture. Once past some 20 seconds of arc from the exciting star, where the radiation field is no longer intense enough to dehydrogenate and fragment the $\mathrm{PAH}$ population, the dominant cation contribution gradually decreases upon moving away from the star, behavior consistent with a decreasing radiation field. There is a slower decrease in $\mathrm{PAH}$ size with distance. A distinct discontinuity in the breakdown maps coincides with the transition between the diffuse PDR medium and the denser cloud material evident in the $H S T$ visible image (Figure 1) and as an abrupt intensity change of the $\mathrm{H}_{2}$ emission lines. The change in the spatial distributions of both small and large PAHs approaching the vicinity of the exciting star suggests active photo-chemistry taking place in this region. Thus, the database breakdown by subclass captures the morphological structure of the region and the change in local conditions.

Particularly interesting are the PAH hydrogen adjacency class ratio maps. Hydrogen adjacency, the number of hydrogens on each peripheral hexagonal ring, is a measure of PAH edge structure and overall shape. $\mathrm{PAH} \mathrm{CH}_{\text {oop }}$ bands tend to fall in spectroscopic ranges characteristic of hydrogen adjacency class and ratios of observed $\mathrm{CH}_{\text {oop }}$ band strengths are generally used to gain insight into the average $\mathrm{PAH}$ edge structure. However, some of these bands overlap, leading to uncertainties in these conclusions. The novel fitting procedure used here overcomes this limitation because hydrogen adjacency class information is inherently part of the results. The hydrogen adjacency class ratio distribution maps also show a bisection along the boundary between the diffuse and denser material as well as finer details into the stepwise PAH dehydrogenation and fragmentation stages approaching both the exciting star and YSOs.

Template spectra are derived for $\mathrm{PAH}$ size and charge. These are consistent with assignments of the different emission components found in the literature, namely that emission in the $5-10 \mu \mathrm{m}$ region is dominated by $\mathrm{PAH}$ cations while emission in the $10-15 \mu \mathrm{m}$ region originates from an evenly split mixture of neutral PAHs and PAH cations; with the caveat that the 6.2 and the $11.0 \mu \mathrm{m}$ bands require PANH cations.

The ability to construct subclass template spectra suggests "principal" emission and PAH population components. Decomposition of the spectral map data using these template spectra provides satisfactory results for only a fraction of the computational cost of the full fit. However, the utility and universal applicability of these template spectra remains to be seen.

Improvement of database fitting techniques lies in (1) the adoption of a more sophisticated emission model, including overtone, combination, and hot bands as well as anharmonic shifts to fully capture their contribution to the observed band profiles and peak positions and (2) the incorporation of the spectra of larger PAHs, PANHs, dehydrogenated PAHs, and fullerenes as well as other relevant (aromatic) species (e.g., VSGs, PAH clusters, HACs CHONS, etc.) to "complete" the database. On the astronomical side, there is a need for complete spectral coverage that includes emission from the $3.3 \mu \mathrm{m}$ and $15-20 \mu \mathrm{m}$ regions along the same lines-of-sight and with the same apertures as the 5-15 $\mu \mathrm{m}$ region. Such an increase in spectral bandwidth significantly increases the spectral information content, thereby providing new, fundamental information that is critically needed to place tight constraints on the family of aromatic species that produce the well-known, ubiquitous, interstellar, mid-IR emission features. 
This work is based on observations made with the Spitzer Space Telescope, which is operated by the Jet Propulsion Laboratory, California Institute of Technology under NASA contract 1407. Support from NASA's Laboratory Astrophysics, "Carbon in the Galaxy" consortium grant (NNH10ZDA001N); and NASA's Astrobiology; Astronomy + Physics Research and Analysis (APRA; NNX07AH02G), and Spitzer Space Telescope Support Programs (50082) are greatly acknowledged. C.B. is especially grateful for appointments to the NASA Postdoctoral Program at the Ames Research Center administered by Oak Ridge Associated Universities through a contract with NASA and by San José State University Research Foundation (grant NNX11AJ33A). Lastly, the careful reading and helpful suggestions made by an anonymous referee are acknowledged.

\section{APPENDIX \\ IDL-CODE}

Below, in Lst. 1, a demonstration of the use of the AmesPAHdbIDLSuite to fit an astronomical spectrum. Similar example code can be found in fit_a_spectrum.pro in the examples directory of the AmesPAHdbIDLSuite. The latest version of the AmesPAHdbIDLSuite can be downloaded from www.astrochem.org/pahdb.

Lst. 1. IDL code snippet illustrating the use of the AmesPAHdbIDLSuite to fit an astronomical spectrum. Similar sample code can be found in fit_a_spectrum.pro in the examples directory of the AmesPAHdbIDLSuite.

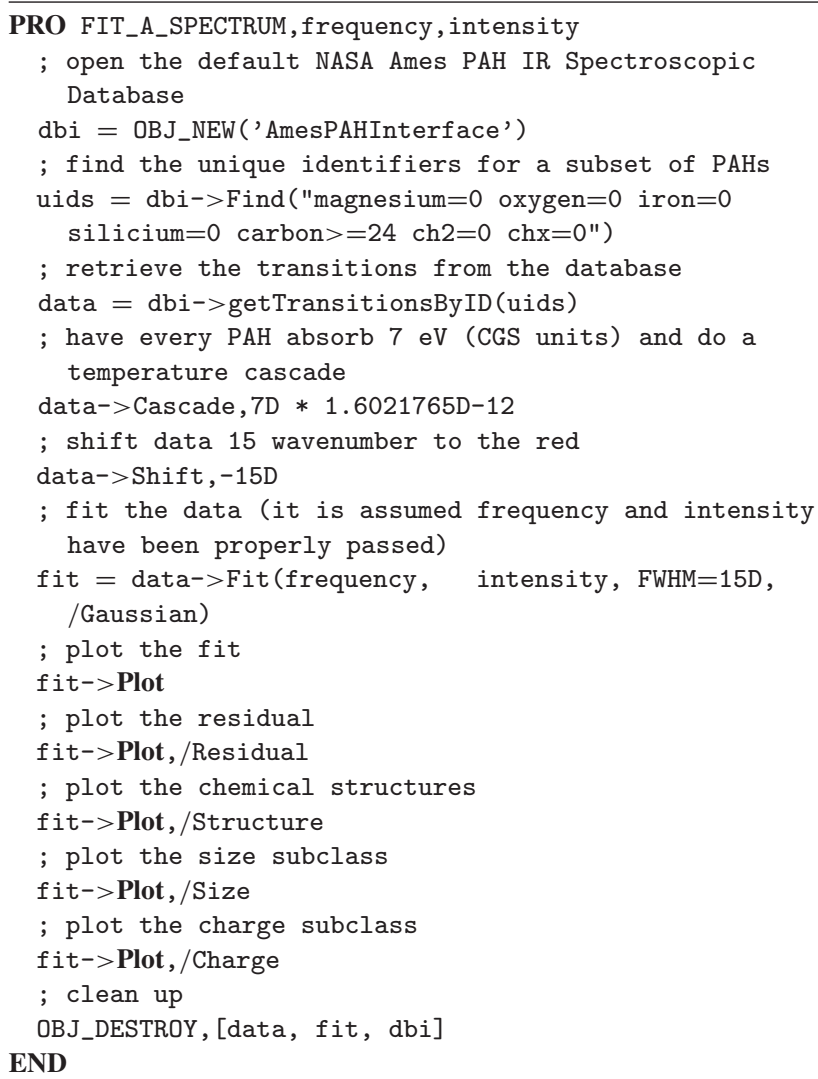

\section{REFERENCES}

Abergel, A., Bernard, J. P., Boulanger, F., et al. 2002, A\&A, 389, 239 Baratta, G., Arena, M., Strazzulla, G., et al. 1996, NIMPB, 116, 195 Barker, J. R., Allamandola, L. J., \& Tielens, A. G. G. M. 1987, ApJL, 315, L61
Bauschlicher, C. W., Boersma, C., Ricca, A., et al. 2010, ApJS, 189, 341 Bauschlicher, C. W., Peeters, E., \& Allamandola, L. J. 2008, ApJ, 678, 316 Bauschlicher, C. W., Peeters, E., \& Allamandola, L. J. 2009, ApJ, 697, 311 Berné, O., Joblin, C., Deville, Y., et al. 2007, A\&A, 469, 575

Berné, O., Mulas, G., \& Joblin, C. 2013, A\&A, 550, L4

Berné, O., \& Tielens, A. G. G. M. 2012, PNAS, 109, 401

Boersma, C., Bauschlicher, C. W., Allamandola, L. J., et al. 2010, A\&A, 511, A32

Boersma, C., Mattioda, A. L., Bauschlicher, C. W., et al. 2009, ApJ, 690, 1208

Boersma, C., Rubin, R. H., \& Allamandola, L. J. 2012, ApJ, 753, 168

Bregman, J., \& Temi, P. 2005, ApJ, 621, 831

Brenner, J., \& Barker, J. R. 1992, ApJL, 388, L39

Cami, J. 2011, in EAS Publications Ser. 46, 117

Cami, J., Bernard-Salas, J., Peeters, E., \& Malek, S. E. 2010, Sci, 329, 1180

Cherchneff, I., \& Barker, J. R. 1989, in IAU Symp. 135, Interstellar Dust, ed. L. J. Allamandola \& A. G. G. M. Tielens (Cambridge: Cambridge Univ. Press), 151

Cohen, M., Allamandola, L., Tielens, A. G. G. M., et al. 1986, ApJ, 302, 737

Colangeli, L., Mennella, V., \& Bussoletti, E. 1992, ApJ, 385, 577

Colangeli, L., Mennella, V., Palumbo, P., Rotundi, A., \& Bussoletti, E. 1995, A\&AS, 113,561

Cook, D. J., \& Saykally, R. J. 1998, ApJ, 493, 793

Disney, M., Davies, J., \& Phillipps, S. 1989, MNRAS, 239, 939

Draine, B. T., \& Li, A. 2007, ApJ, 657, 810

Dwek, E., Arendt, R. G., Fixsen, D. J., et al. 1997, ApJ, 475, 565

Fleming, B., France, K., Lupu, R. E., \& McCandliss, S. R. 2010, ApJ, 725, 159

Flickinger, G. C., \& Wdowiak, T. J. 1990, ApJL, 362, L71

Flickinger, G. C., Wdowiak, T. J., \& Gomez, P. L. 1991, ApJL, 380, L43

Furton, D. G., Laiho, J. W., \& Witt, A. N. 1999, ApJ, 526, 752

Geballe, T. R., Lacy, J. H., Persson, S. E., McGregor, P. J., \& Soifer, B. T. 1985, ApJ, 292, 500

Gillett, F. C., Forrest, W. J., \& Merrill, K. M. 1973, ApJL, 184, L93

Hony, S., van Kerckhoven, C., Peeters, E., et al. 2001, A\&A, 370, 1030

Houck, J. R., Roellig, T. L., van Cleve, J., et al. 2004, ApJS, 154, 18

Hu, A., \& Duley, W. W. 2007, ApJL, 660, L137

Hudgins, D. M., \& Allamandola, L. J. 1999, ApJL, 516, L41

Hudgins, D. M., Bauschlicher, C. W., \& Allamandola, L. J. 2005, ApJ, 632,316

Joblin, C., Boissel, P., Leger, A., D’Hendecourt, L., \& Defourneau, D. 1995 A\&A, 299, 835

Jones, A. P. 2012a, A\&A, 540, A1

Jones, A. P. 2012b, A\&A, 540, A2

Jones, A. P. 2012c, A\&A, 542, A98

Landsman, W. B. 1993, in ASP Conf. Ser. 52, Astronomical Data Analysis Software and Systems II, ed. R. J. Hanisch, R. J. V. Brissenden, \& J. Barnes (San Francisco, CA: ASP), 246

Lawson, C. L., \& Hanson, R. J. 1974, Solving Least Squares Problems (PrenticeHall Series in Automatic Computation; Englewood Cliffs, NJ: Prentice-Hall)

Lee, W., \& Wdowiak, T. J. 1993, ApJL, 417, L49

Mattioda, A. L., Allamandola, L. J., \& Hudgins, D. M. 2005, ApJ, 629, 1183

Mennella, V., Baratta, G. A., Esposito, A., Ferini, G., \& Pendleton, Y. J. 2003, ApJ, 587, 727

Mennella, V., Brucato, J. R., Colangeli, L., \& Palumbo, P. 1999, ApJL, 524, L71

Montillaud, J., Joblin, C., \& Toublanc, D. 2013, A\&A, 552, A15

Morterra, C., \& Low, M. 1983, Carbon, 21, 283

Moutou, C., Sellgren, K., Leger, A., et al. 1998, in ASP Conf. Ser. 132, Star Formation with the Infrared Space Observatory, ed. J. Yun \& L. Liseau (San Francisco, CA: ASP), 47

Pech, C., Joblin, C., \& Boissel, P. 2002, A\&A, 388, 639

Peeters, E., Hony, S., van Kerckhoven, C., et al. 2002, A\&A, 390, 1089

Pilleri, P., Montillaud, J., Berné, O., \& Joblin, C. 2012, A\&A, 542, A69

Rapacioli, M., Joblin, C., \& Boissel, P. 2005, A\&A, 429, 193

Ricca, A., Bauschlicher, C. W., Jr., Boersma, C., Tielens, A. G. G. M., \& Allamandola, L. J. 2012, ApJ, 754, 75

Rieke, G. H., \& Lebofsky, M. J. 1985, ApJ, 288, 618

Rosenberg, M. J. F., Berné, O., Boersma, C., Allamandola, L. J., \& Tielens, A. G. G. M. 2011, A\&A, 532, A128

Schnaiter, M., Mutschke, H., Dorschner, J., Henning, T., \& Salama, F. 1998, ApJ, 498, 486

Sellgren, K. 1983, AJ, 88, 985

Sellgren, K. 1984, ApJ, 277, 623

Sellgren, K., Uchida, K. I., \& Werner, M. W. 2007, ApJ, 659, 1338

Sellgren, K., Werner, M. W., Ingalls, J. G., et al. 2010, ApJL, 722, L54

Smith, J. D. T., Armus, L., Dale, D. A., et al. 2007a, PASP, 119, 1133 
Smith, J. D. T., Draine, B. T., Dale, D. A., et al. 2007b, ApJ, 656, 770

Tielens, A. G. G. M. 2008, ARA\&A, 45, 289

van den Ancker, M. E., The, P. S., Tjin A Djie, H. R. E., et al. 1997, A\&A, 324, L33

van Diedenhoven, B., Peeters, E., van Kerckhoven, C., et al. 2004, ApJ, 611,928
Verstraete, L., Pech, C., Moutou, C., et al. 2001, A\&A, 372, 981

Weingartner, J. C., \& Draine, B. T. 2001, ApJ, 548, 296

Werner, M. W., Roellig, T. L., Low, F. J., et al. 2004a, ApJS, 154,

Werner, M. W., Uchida, K. I., Sellgren, K., et al. 2004b, ApJS, 154, 309

Williams, R. M., \& Leone, S. R. 1995, ApJ, 443, 675

Witt, A. N., Gordon, K. D., Vijh, U. P., et al. 2006, ApJ, 636, 303 